\title{
Different-case repetition still leads to perceptual blindness
}

\author{
KATHLEEN M. MAROHN and LARRY HOCHHAUS \\ Oklahoma State University, Stillwater, Oklahoma
}

\begin{abstract}
Jacoby and Dallas (1981) were the first to label the perceptual enhancement of a stimulus due to a prior presentation as "relative perceptual fluency." Our earlier work (Marohn \& Hochhaus, in press) demonstrated that consecutive repetition priming and consecutive semantic priming have different effects on perceptual fluency, wherein repetition priming causes a phenomenon we call "perceptual blindness," in which the subject fails to perceive the second presentation. Is this failure simply a result of the exact physical repetition of the priming stimulus? The present experiment was a follow-up study designed to ascertain what effect a change in letter case format would have on the perceptual fluency of the repeated target word. Repetition in a different letter case showed that this "cognitive refractoriness" generalizes to different test conditions and goes beyond the physical features of the letters to the word as a whole unit.
\end{abstract}

Jacoby and Dallas (1981) introduced the informationprocessing mechanism of "relative perceptual fluency." Perceptual fluency represents a perceptual enhancement of a stimulus upon recognition testing as a consequence of prior study of that stimulus. Witherspoon and Allan (1985) employed quantitative measures for assessing perceptual fluency. They found longer duration judgments and greater identification accuracy for previously studied words than for new words. These authors believed that it is familiarity, caused by a prior presentation, that explains the longer perceived durations and the increased accuracy in word recognition.

Our earlier work (Marohn \& Hochhaus, in press) documented the phenomenon of relative perceptual fluency for consecutive semantic priming. Our method involved sequential computer presentation under three conditions: unrelated pairing, semantic priming, and repetition priming. Our subjects reported significantly longer durations and greater accuracy for the semantic condition. Originally, we included consecutive repetition priming as a reference point for the semantic condition. On the basis of the results of Witherspoon and Allan (1985) and research concerning repetition and word identification (Forbach, Stanners, \& Hochhaus, 1974; Forster \& Davis, 1984), we expected repetition priming to produce stronger effects on perceptual fluency than those anticipated for semantic priming. This, however, was not the case. Durations of repeated items were judged to be significantly shorter than durations of unrelated targets. In addition, word identification for repeated words was significantly poorer than that for unrelated or semantic targets.

This research was supported in part by a grant from Oklahoma State University to the second author. Requests for reprints should be sent to Larry Hochhaus, Department of Psychology, 215 North Murray, Oklahoma State University, Stillwater, OK 74078.
Thus, contrary to our predictions, consecutive semantic priming and consecutive repetition priming had different effects on relative perceptual fluency. Why this happens is not immediately clear. Perhaps the two priming processes affect separate stages of word recognition, as the work of den Heyer, Goring, and Dannenbring (1985) suggests. Or are shorter apparent durations and decreased identification accuracy for successive repetition simply a result of the exact physical repetition of the priming stimulus? The repetition of words in various letter case formats appears to be an appropriate method for finding an answer to this question (Forster \& Davis, 1984).

As in our prior study, in the present experiment we employed two independent variables. One involved the presentation of pairs of words in two conditions: unrelated and repetition. The second independent variable was the actual duration of presented words. Two dependent variables were measured: judged durations and accuracy in word identification. The priming word was in lowercase letters and the target word in uppercase letters. This change in letter case format was expected to enhance the subject's perception of the repeated target and to produce the longer perceived durations (and greater identification a. Iracy) associated with perceptual fluency.

\section{METHOD}

Subjects

Eighteen Oklahoma State University student volunteers participated in this experiment. They were given a small amount of class credit for their efforts.

\section{Materials \\ A word pool of 132 associated pairs was selected from The Connec- ticut Free Associational Norms (Bousfield, Cohen, Whitmarsh, \& Kin- cade, 1961). The mean association value for the 120 test pairs was 77.18 (range 45-136). We selected the 120 most highly associated pairs for the experimental test. Thus the mean association value for the 12 prac- tice pairs was only 41.17 (range 39-44). Practice and test words were}


paired under two conditions: (1) unrelated-priming word paired with an unrelated word, and (2) repetition-priming word paired with itself. The unrelated pairs were created by randomly assigning the response members of 44 of the associated pairs to new stimulus terms within that set of 44 pairs. Repeated pairs were created by replacing the response member of a second random subset of 44 of the associated pairs with the stimulus member of that pair.

An Apple IIc computer was used to present the words. The priming words were in lowercase letters and the target words were in uppercase letters. The mean length of words was 4.41 letters (range 2-8 letters). The visual angles subtended by each letter were $.56^{\circ}$ for height and $.32^{\circ}$ for width. The viewing distance was approximately $63.5 \mathrm{~cm}$.

\section{Procedure}

The subjects were tested individually. They sat directly in front of the computer screen while the words flashed on the screen. The subjects read instructions defining their task in each phase of testing. A session lasted approximatelay $30 \mathrm{~min}$ and consisted of two phases.

Phase 1: Pretraining for time judgments. A trial began when the message "Press Return When Ready" appeared on the screen. When the subject pressed the "return"' key, the message was erased and two horizontal $1.75-\mathrm{cm}$ lines (with $4.76 \mathrm{~mm}$ vertical separation) appeared and remained as a marker for $500 \mathrm{msec}$. The lines oriented the subjects to the location where the string of five characters (ZZZZZ) would next appear. The string of $\mathrm{Zs}$ was presented for durations of $20,160,300$, and $440 \mathrm{msec}$. The software clock of Deiner and Smee (1984) was used, but because we used an Apple IIc computer, presentations could not be synchronized with the raster scanner; hence these display times and others mentioned throughout this paper are only approximations.

The times employed in Phase 1 were chosen on the basis of a pilot study so that, on average, the subjects were performing at about $50 \%$ correct. A score of $25 \%$ is chance in this task. The task for this phase was to categorize each duration by pressing $1,2,3$, or 4 on the computer keyboard, with 1 representing the shortest presentation and 4 the longest presentation. There were 100 trials, with each of the four durations occurring 25 times in random order.

Phase 2: Test. The subjects read instructions indicating that they were to identify the target word and to judge the duration of its presentation on the screen. A test trial began when the message "Press Space Bar When Ready"' appeared. Then the priming word was presented between two horizontal $1.75-\mathrm{cm}$ lines for $500 \mathrm{msec}$. After each priming word was presented, the target word appeared two lines lower, also in the center of the screen, for either 16 or $32 \mathrm{msec}$ in a random order. Durations of 16 and $32 \mathrm{msec}$ were chosen on the basis of our pilot studies so that subjects would have less than $100 \%$ accuracy in word identification. A 1-sec mask of five ampersands followed the target presentation. Phase 2 involved the two presentation times combined factorially with the two pair conditions, unrelated and repetition. There were 80 presentation trials of paired words, consisting of 20 instances of each of the four combinations of conditions. The order of the 80 trials was random. The subject was requested to identify the target word by saying it aloud, and then to judge the duration of the presentation of the target word, using the four time categories of the pretraining phase.

\section{RESULTS}

The results of Phase 1 were consistent with those of our previous experiments $(M=57.44$ correct responses for Phase 1). Thus, the subjects were able to categorize the durations at about $50 \%$ correct.

The reported data are from Phase 2 . The judged duration data were analyzed with a $2 \times 2$ repeated measures analysis of variance. The factors were duration (16 and $32 \mathrm{msec}$ ) and pair type (unrelated and repetition). The means of ratings on the 4-point scale were 1.88 (unrelated) and 1.67 (repetition). The difference between the two conditions is significant $[F(1,17)=18.60, p<.05]$. There was no interaction between duration and pair type $[F(1,17)$ $=1.17, p>.05]$. The difference between short and long durations was constant for each of the two pairing conditions.

The subjects judged the two presentation times according to their appropriate short or long category. The mean judged durations for each presentation length (16 and $32 \mathrm{msec}$ ) were 1.63 (short) and 1.92 (long). This is a significant difference $[F(1,17)=22.98, p<.05]$.

Of total errors ( 29.22 for the 80 presentations), the repetition condition evoked the most $(17.83$, or $61 \%)$, whereas the unrelated condition evoked only $11.83(39 \%)$. This difference is significant $[F(1,17)=6.03, p<.05]$. Error means for the two presentation durations were 18.61 (short) and 10.61 (long), a significant difference $[F(1,17)$ $=42.39, p<.05]$. The interaction between duration and pair type is nonsignificant $[F(1,17)=0.74, p>.05]$; that is, the difference between short and long durations was constant for the two pairing conditions. Omissions were the most frequent type of errors $(71 \%)$.

\section{DISCUSSION}

The change in letter case format was not effective in eliciting relative perceptual fluency in the repetition condition. The alteration of the physical representation of the repeated stimulus was not sufficient to produce longer perceived durations or increased accuracy in word identification, as occurs for semantic priming. Both conditions produced the same reduction in apparent duration and identification accuracy as in our earlier studies. On the basis of these results, we conclude that there is more to the explanation of our earlier results than the mere fact that physical features affect processing of the second item. Even with the change in letter case format, the target word continues to bypass the necessary processing for word identification and leads to shorter judged durations and decreased identification accuracy. Apparently, consecutive repetition priming causes a phenomenon analogous to "perceptual blindness," the logical opposite of perceptual fluency.

This experiment shows us that the surprising effects of consecutive repetition priming are not a consequence of the physical similarity between the priming word and the target word. Now our investigation into this paradox can continue in at least two directions. The first effort would involve a manipulation of the number of intervening items between repetitions in a manner similar to that of McKoon and Ratcliff (1986). Thus, the repeated word would never immediately follow its first presentation as a priming word, as it has in our work thus far. It is to be hoped that this separation in time and distance would lead to a repetition effect similar to that found in a spaced study list (Cuddy \& Jacoby, 1982; Jacoby, 1978; Jacoby \& Dallas, 1981). Perhaps a measure of forgetting would occur and force the subject to reprocess the repeated target word (Rose, 1984). This forgetting does not occur in our consecutive repetition priming process. Another approach to documenting perceptual fluency using our method would be to manipulate the duration interval between the presentation of the priming word and the presentation of the target word. A parametric study of these different duration intervals and an analysis of the effect of stimulus onset asynchrony might reveal the time course of "cognitive refractoriness."

\section{REFERENCES}

Bousfield, W. A., Cohen, B. H., Whitmarsh, G. A., \& Kincade, W. D. (1961). The Connecticut free associational norms (Tech. Rep. No. 35). Storrs: University of Connecticut.

CuDDY, L. J., \& JACOBY, L. L. (1982). When forgetting helps memory: An analysis of repetition effects. Journal of Verbal Learning \& Verbal Behavior, 21, 451-467. 
Deiner, D., \& Smee, W. P. (1984). Apple tachistoscope. Behavior Research Methods, Instruments, \& Computers, 16, 540-544.

den Heyer, K., Goring, A., \& Dannenbring, G. L. (1985). Semantic priming and word repetition: The two effects are additive. Journal of Memory \& Language, 24, 699-716.

Forbach, G. B., Stanners, R. F., \& Hochhaus, L. (1974). Repetition and practice effects in a lexical decision task. Memory \& Cognition, 2, 332-339.

Forster, K. I., \& DAVIS, C. (1984). Repetition priming and frequency attentuation in lexical access. Journal of Experimental Psychology: Learning, Memory, \& Cognition, 10, 680-689.

JACOBY, L. L. (1978). On interpreting the effects of repetition: Solving a problem versus remembering a solution. Journal of Verbal Learning \& Verbal Behavior, 17, 649-667.

JACOBY, L. L., \& DallaS, M. (1981). On the relationship between autobiographical memory and perceptual learning. Journal of Experimental Psychology: General, 110, 306-340.

Marohn, K. M., \& Hochhaus, L. (in press). Semantic priming increases and repetition priming decreases apparent stimulus duration. Journal of General Psychology.

McKoon, G., \& RATCLIF, R. (1986). Automatic activation of episodic information in a semantic memory task. Journal of Experimental Psychology: Learning, Memory, \& Cognition, 12, 108-115.

Rose, R. J. (1984). Processing time for repetitions and the spacing effect. Canadian Journal of Psychology, 83, 537-550.

WithersPoON, D., \& AllaN, L. G. (1985). The effect of a prior presentation on temporal judgments in a perceptual identification task. Memory \& Cognition, 13, 101-111.

(Manuscript received for publication June 5, 1987.) 